Bull. Korean Math. Soc. 49 (2012), No. 1, pp. 145-154

http://dx.doi.org/10.4134/BKMS.2012.49.1.145

\title{
THE LINEAR 2-ARBORICITY OF PLANAR GRAPHS WITHOUT ADJACENT SHORT CYCLES
}

\author{
Hong-Yu Chen, Xiang Tan, and Jian-Liang Wu
}

\begin{abstract}
Let $G$ be a planar graph with maximum degree $\Delta$. The linear 2-arboricity $l a_{2}(G)$ of $G$ is the least integer $k$ such that $G$ can be partitioned into $k$ edge-disjoint forests, whose component trees are paths of length at most 2. In this paper, we prove that (1) $l a_{2}(G) \leq\left\lceil\frac{\Delta}{2}\right\rceil+8$ if $G$ has no adjacent 3-cycles; (2) $l a_{2}(G) \leq\left\lceil\frac{\Delta}{2}\right\rceil+10$ if $G$ has no adjacent 4 -cycles; (3) $l a_{2}(G) \leq\left\lceil\frac{\Delta}{2}\right\rceil+6$ if any 3 -cycle is not adjacent to a 4 -cycle of $G$.
\end{abstract}

\section{Introduction}

In this paper, all graphs are finite, simple and undirected. For a real number $x,\lceil x\rceil$ is the least integer not less than $x$ and $\lfloor x\rfloor$ is the largest integer not larger than $x$. Let $G$ be a graph. We use $V(G)$ and $E(G)$ to denote the vertex set and the edge set, respectively. If $u v \in E(G)$, then $u$ is said to be the neighbor of $v$, and $N(v)$ is the set of neighbors of $v$. The degree of a vertex $v d(v)=|N(v)|$, $\delta(G)$ is the minimum degree and $\Delta(G)$ is the maximum degree of $G$. A $k-, k^{+}-$ or $k^{-}$- vertex is a vertex of degree $k$, at least $k$, or at most $k$, respectively. A $k$ - cycle is a cycle of length $k$. Two cycles are said to be adjacent if they are incident with a common edge. For $s \geq 2$, an even cycle $C=v_{1} v_{2} \cdots v_{2 s} v_{1}$ is called a 2-alternating cycle if $d\left(v_{1}\right)=\bar{d}\left(v_{3}\right)=\cdots=d\left(v_{2 s-1}\right)=2$.

An edge-partition of a graph $G$ is a decomposition of $G$ into subgraphs $G_{1}, G_{2}, \ldots, G_{m}$ such that $E(G)=E\left(G_{1}\right) \cup E\left(G_{2}\right) \cup \cdots \cup E\left(G_{m}\right)$ and $E\left(G_{i}\right) \cap$ $E\left(G_{j}\right)=\emptyset$ for $i \neq j$. A linear $k$-forest is a graph in which each component is a path of length at most $k$. The linear $k$-arboricity $l a_{k}(G)$ of a graph $G$ is the least integer $m$ such that $G$ can be edge-partitioned into $m$ linear $k$-forests. Clearly, $l a_{k}(G) \geq l a_{k+1}(G)$ for any $k \geq 1$. For extremities, $l a_{1}(G)$ is the edge chromatic number $\chi^{\prime}(G)$ of $G ; l a_{\infty}(G)$ representing the case when component paths have unlimited lengths is the ordinary linear arboricity $l a(G)$ of $G$.

Received September 13, 2010; Revised May 21, 2011.

2010 Mathematics Subject Classification. 05C15.

Key words and phrases. planar graph, linear 2-arboricity, cycle.

This work was partially supported by grants 10971121 and 60873207 from NSFC and by grants Y52012-20 from Scientific research Foundation for Talent Introduction of Shanghai Institute of Technology. 
The linear $k$-arboricity of a graph was first introduced by Habib and Péroche [7]. They posed the following conjecture.

Conjecture A. For a graph $G$ of order $n$ and a positive integer $i$,

$$
l a_{i}(G) \leq\left\{\begin{array}{l}
\left\lceil(\Delta n+1) / 2\left\lfloor\frac{i n}{i+1}\right\rfloor\right\rceil \text { if } \Delta \neq n-1 \\
\left\lceil(\Delta n) / 2\left\lfloor\frac{i n}{i+1}\right\rfloor\right\rceil \text { if } \Delta=n-1 .
\end{array}\right.
$$

The linear $k$-arboricity of cycles, trees, complete graphs and complete bipartite graphs has been determined in [5], [6]. Thomassen [12] proved that $l a_{k} \leq 2$ for a cubic graph $G$, where $k \geq 5$, and this result is best possible. Chang [3] and Chang et al. [4] investigated the algorithmic aspects of the linear $k$-arboricity. It was further studied by Bermond et al. [2], Jackson and Wormald [8], and Aldred and Wormald [1]. Lih, Tong and Wang [9] proved that for a planar graph $G, l a_{2}(G) \leq\left\lceil\frac{\Delta+1}{2}\right\rceil+12$; Moreover, $l a_{2}(G) \leq\left\lceil\frac{\Delta+1}{2}\right\rceil+6$ if $G$ does not contain 3-cycles. Qian and Wang [11] proved that for a planar graph $G$ without 4-cycles, $l a_{2}(G) \leq\left\lceil\frac{\Delta+1}{2}\right\rceil+3$. $\mathrm{Ma}$, $\mathrm{Wu}$ and $\mathrm{Yu}[10]$ proved that for a planar graph $G$ without 5 - or 6 -cycles, $l a_{2}(G) \leq\left\lceil\frac{\Delta+1}{2}\right\rceil+6$. For a planar graph $G$, we will prove that $(1) l a_{2}(G) \leq\left\lceil\frac{\Delta}{2}\right\rceil+8$ if $G$ has no adjacent 3-cycles; (2) $l a_{2}(G) \leq\left\lceil\frac{\Delta}{2}\right\rceil+10$ if $G$ has no adjacent 4-cycles; (3) $l a_{2}(G) \leq\left\lceil\frac{\Delta}{2}\right\rceil+6$ if any 3 -cycle is not adjacent to a 4 -cycle of $G$.

\section{Main results and their proofs}

In the section, we always assume that a planar graph $G$ has always been embedded in the plane. Let $G$ be a planar graph and $F(G)$ be the face set of $G$. For $f \in F(G)$, the degree of $f$, denoted by $d(f)$, is the number of edges incident with it, where each cut-edge is counted twice. A $k-, k^{+}$- or $k^{-}$- face is a face of degree $k$, at least $k$, or at most $k$, respectively. Let $n_{i}(v)$ denote the number of $i$-vertices of $G$ adjacent to the vertex $v, q_{i}(v)$ the number of $i$-faces of $G$ incident with $v$. A $k$-face with consecutive vertices $v_{1}, v_{2}, \ldots, v_{k}$ along its boundary in some direction is often said to be $\left(d\left(v_{1}\right), d\left(v_{2}\right), \ldots, d\left(v_{k}\right)\right)$-face.

Lemma 1. Let $G$ be a connected planar graph with $\delta(G) \geq 2$. If $G$ has no adjacent 3-cycles, then $G$ contains an edge $x y$ such that $d(x)+d(y) \leq 11$, or $G$ contains a 2-alternating cycle.

Proof. Suppose, to the contrary, that $G$ is such a connected planar graph not satisfying the lemma. Then we have

(a) For any vertex $v, q_{3}(v) \leq\left\lfloor\frac{d(v)}{2}\right\rfloor$;

(b) For any vertex $v, n_{2}(v)+n_{3}(v)+q_{3}(v) \leq d(v)$;

(c) Let $G_{2}$ be the subgraph induced by the edges incident with the 2 -vertices of $G$, then $G_{2}$ is a forest and there exists a matching $M$ such that all 2-vertices in $G_{2}$ are saturated.

(a) is obvious. For (b), suppose $f$ is a 3 -face incident with $v$. Since $d(x)+$ $d(y) \geq 12$ for any edge $x y \in E(G), f$ is incident with at most one $5^{-}$-vertex. 
So $v$ is adjacent to at least $q_{3}(v) 6^{+}$-vertices. Hence, $d(v)-n_{2}(v)-n_{3}(v) \geq$ $d(v)-\sum_{i=2}^{5} n_{i}(v) \geq q_{3}(v)$.

For (c), since $d(x)+d(y) \geq 12$ for every edge $x y \in E(G)$, every pair of 2 -vertices is nonadjacent. Hence, $G_{2}$ does not contain any odd cycle. Since $G$ does not contain any 2 -alternating cycle, $G_{2}$ does not contain any even cycle. So every component of $G_{2}$ is a tree and there exists a matching $M$ such that all 2-vertices in $G_{2}$ are saturated.

If $u v \in M$ and $d(u)=2$, we call $v$ the 2-master of $u$.

By Euler's formula $|V|-|E|+|F|=2$, we have

$$
\sum_{v \in V}(d(v)-4)+\sum_{f \in F}(d(f)-4)=-4(|V|-|E|+|F|)=-8<0 .
$$

We define $c h$ to be the initial charge. Let $\operatorname{ch}(x)=d(x)-4$ for each $x \in$ $V(G) \cup F(G)$. In the following, we will reassign a new charge denoted by $\operatorname{ch}^{\prime}(x)$ to each $x \in V(G) \cup F(G)$ according to the discharging rules. Since our rules only move charges around, and do not affect the sum, we have

$$
\sum_{x \in V(G) \cup F(G)} \operatorname{ch}^{\prime}(x)=\sum_{x \in V(G) \cup F(G)} \operatorname{ch}(x)=-8 .
$$

In the following, we will show that $\operatorname{ch}^{\prime}(x) \geq 0$ for each $x \in V(G) \cup F(G)$, a contradiction to (2), completing the proof.

The discharging rules are defined as follows.

R1-1. Each 2-vertex receives 2 from its 2-master.

R1-2. Each 3 -vertex receives $\frac{1}{3}$ from each of its neighbors.

R1-3. If a 3 -face $f$ is incident with a $4^{-}$-vertex, then $f$ receives $\frac{1}{2}$ from each of another two incident vertices; Otherwise, $f$ receives $\frac{1}{3}$ from each of its incident vertices.

Let $f$ be a face of $G$. If $d(f) \geq 4$, then $\operatorname{ch}^{\prime}(f)=c h(f) \geq 0$. If $d(f)=3$, then it is incident with at most one $4^{-}$-vertex. It follows that $\operatorname{ch}^{\prime}(f) \geq \operatorname{ch}(f)+$ $\min \left\{2 \times \frac{1}{2}, 3 \times \frac{1}{3}\right\}=0$ by R1-3.

Let $v$ be a vertex of $G$. If $d(v)=2$, then $\operatorname{ch}^{\prime}(v)=\operatorname{ch}(v)+2=0$ by R1-1. If $d(v)=3$, then $\operatorname{ch}^{\prime}(v)=\operatorname{ch}(v)+3 \times \frac{1}{3}=0$ by R1-2. If $d(v)=4$, then $\operatorname{ch}^{\prime}(v)=\operatorname{ch}(v)=d(v)-4=0$. If $5 \leq d(v) \leq 8$, then $q_{3}(v) \leq\left\lfloor\frac{d(v)}{2}\right\rfloor$ by (a), it follows that $\operatorname{ch}^{\prime}(v) \geq \operatorname{ch}(v)-\frac{1}{2} q_{3}(v) \geq 0$ by R1-3. If $d(v)=9$, then $q_{3}(v) \leq 4$ by $(\mathrm{a})$, and $n_{3}(v) \leq d(v)-q_{3}(v)$ by (b). It follows that $\operatorname{ch}^{\prime}(v) \geq$ $\operatorname{ch}(v)-\frac{1}{2} q_{3}(v)-\frac{1}{3} n_{3}(v) \geq 0$ by R1-2 and R1-3. If $d(v) \geq 10$, then $q_{3}(v) \leq\left\lfloor\frac{d(v)}{2}\right\rfloor$ by $(\mathrm{a})$, and $n_{3}(v) \leq d(v)-q_{3}(v)-n_{2}(v)$ by (b). It follows that $\operatorname{ch}^{\prime}(v) \geq$ $\operatorname{ch}(v)-\max \left\{2+\frac{1}{2} q_{3}(v)+\frac{1}{3}\left(d(v)-q_{3}(v)-n_{2}(v)\right), \frac{1}{2} q_{3}(v)+\frac{1}{3}\left(d(v)-q_{3}(v)\right)\right\} \geq$ 
$\max \left\{2+\frac{1}{2} q_{3}(v)+\frac{1}{3}\left(d(v)-q_{3}(v)-1\right), \frac{1}{2} q_{3}(v)+\frac{1}{3}\left(d(v)-q_{3}(v)\right)\right\} \geq 0$ by R1-1, R1-2 and R1-3.

Hence we complete the proof of the lemma.

Lemma 2. Every planar graph $G$ without adjacent 3-cycles has an edge-partition into two forests $T_{1}, T_{2}$ and a subgraph $H$ such that for every $v \in V(G)$, $d_{T_{1}}(v) \leq\left\lceil\frac{d_{G}(v)}{2}\right\rceil, d_{T_{2}}(v) \leq\left\lceil\frac{d_{G}(v)}{2}\right\rceil$ and $d_{H}(v) \leq 6$.

Proof. The proof of the lemma is by induction on the number $|V(G)|+|E(G)|$. For a planar graph $G$ with $|V(G)|+|E(G)| \leq 5$, the lemma holds obviously. For a planar graph $G$ with $|V(G)|+|E(G)| \geq 6$, if $\Delta(G) \leq 6$, then let $H=G$ and $T_{1}=T_{2}=\emptyset$, the result holds.

Suppose that $\Delta(G) \geq 7$. We may assume that $G$ is a connected planar graph. By the induction, if $G^{\prime}$ is a proper subgraph of $G$, the lemma is true for the graph $G^{\prime}$, that is, $G^{\prime}$ has an edge-partition into two forests $T_{1}^{\prime}, T_{2}^{\prime}$ and a subgraph $H^{\prime}$ such that for every $v \in V\left(G^{\prime}\right), d_{H^{\prime}}(v) \leq 6$ and $d_{T_{i}^{\prime}}(v) \leq\left\lceil\frac{d_{G^{\prime}}(v)}{2}\right\rceil$ for $i=1,2$. We will choose an appropriate subgraph $G^{\prime}$ to extend $T_{1}^{\prime} \cup T_{2}^{\prime} \cup H^{\prime}$ to an edge-partition $T_{1} \cup T_{2} \cup H$ of $G$ satisfying the lemma.

We now consider two cases according to the minimum degree of $G$.

Case 1. $\delta(G)=1$. Let $u v \in E(G)$ and $d_{G}(u)=1$. Define the graph $G^{\prime}=G-u v$.

If $d_{H^{\prime}}(v) \leq 5$, then let $H=H^{\prime}+u v$ and $T_{i}=T_{i}^{\prime}$ for $i=1,2$. It is easy to see that the result holds.

If $d_{H^{\prime}}(v)=6$, suppose that $d_{T_{1}^{\prime}}(v) \leq d_{T_{2}^{\prime}}(v)$. Since $d_{G^{\prime}}(v)=d_{T_{1}^{\prime}}(v)+$ $d_{T_{2}^{\prime}}(v)+d_{H^{\prime}}(v)=d_{T_{1}^{\prime}}(v)+d_{T_{2}^{\prime}}(v)+6$ and $d_{G^{\prime}}(v)=d_{G}(v)-1$, we have $d_{T_{1}^{\prime}}(v) \leq \frac{d_{G}(v)-7}{2}$. Let $T_{1}=T_{1}^{\prime}+u v, T_{2}=T_{2}^{\prime}$ and $H=H^{\prime}$. Thus $d_{T_{2}}(x)=$ $d_{T_{2}^{\prime}}(x)$ and $d_{H}(x)=d_{H^{\prime}}(x)$ for all $x \in V\left(G^{\prime}\right)$. Moreover, $d_{T_{1}}(u)=1=$ $\left\lceil\frac{d_{G}(u)}{2}\right\rceil, d_{T_{1}}(v)=1+d_{T_{1}^{\prime}}(v) \leq 1+\frac{d_{G}(v)-7}{2}<\left\lceil\frac{d_{G}(v)}{2}\right\rceil$, and $d_{T_{1}}(x)=d_{T_{1}^{\prime}}(x)$ for all $x \in V(G) \backslash\{u, v\}$.

Case 2. $\delta(G) \geq 2$. By Lemma 1 , we only need to consider two subcases.

Subcase 1. $G$ contains an edge $x y \in E(G)$ such that $d_{G}(x)+d_{G}(y) \leq 11$.

Define the graph $G^{\prime}=G-x y$ and assume that $d_{H^{\prime}}(x) \leq d_{H^{\prime}}(y)$. If $d_{H^{\prime}}(y) \leq$ 5 , let $H=H^{\prime}+x y, T_{1}=T_{1}^{\prime}$ and $T_{2}=T_{2}^{\prime}$, then the lemma holds obviously.

Suppose that $d_{H^{\prime}}(y)=6$. Then $1 \leq d_{G^{\prime}}(x) \leq 3$ and $d_{T_{1}^{\prime}}(y)+d_{T_{2}^{\prime}}(y)+$ $d_{G^{\prime}}(x) \leq 3$. We may assume $d_{T_{1}^{\prime}}(x) \leq d_{T_{2}^{\prime}}(x)$.

If $d_{G^{\prime}}(x)=3$, then $y \notin V\left(T_{1}^{\prime}\right)$ and $y \notin V\left(T_{2}^{\prime}\right)$. Let $T_{1}=T_{1}^{\prime}+x y, T_{2}=T_{2}^{\prime}$ and $H=H^{\prime}$. If $d_{G^{\prime}}(x)=2$, then $x \in V\left(T_{1}^{\prime}\right)$ and $x \in V\left(T_{2}^{\prime}\right)$ since $d_{T_{i}^{\prime}}(x) \leq$ $\left\lceil\frac{d_{G^{\prime}}(x)}{2}\right\rceil$ for $i=1,2$. Also note that $y \notin V\left(T_{1}^{\prime}\right)$ or $y \notin V\left(T_{2}^{\prime}\right)$, Assume that $y \notin V\left(T_{1}^{\prime}\right)$. Again let $T_{1}=T_{1}^{\prime}+x y, T_{2}=T_{2}^{\prime}$ and $H=H^{\prime}$. We see that $T_{1}$ is a forest and $d_{T_{1}}(x)=2=\left\lceil\frac{3}{2}\right\rceil=\left\lceil\frac{d_{G}(x)}{2}\right\rceil$. If $d_{G^{\prime}}(x)=1$, then $x \notin V\left(T_{1}^{\prime}\right)$. Let $T_{1}=T_{1}^{\prime}+x y, T_{2}=T_{2}^{\prime}$ and $H=H^{\prime}$. We see that $T_{1}$ is a forest and 
$d_{T_{1}}(x)=1=\left\lceil\frac{d_{G}(x)}{2}\right\rceil$. Furthermore, $d_{T_{1}}(y)=d_{T_{1}^{\prime}}(y)+1 \leq 3<\left\lceil\frac{d_{G}(y)}{2}\right\rceil$, the result holds.

Subcase 2. $G$ contains a 2-alternating cycle $C=v_{1} v_{2} \cdots v_{2 s} v_{1}, s \geq 2$, such that $d_{G}\left(v_{1}\right)=d_{G}\left(v_{3}\right)=\cdots=d_{G}\left(v_{2 s-1}\right)=2$.

Define the graph $G^{\prime}=G-E(C)$. Let $T_{1}=T_{1}^{\prime}+\left\{v_{1} v_{2}, v_{3} v_{4}, \ldots, v_{2 s-1} v_{2 s}\right\}$, $T_{2}=T_{2}^{\prime}+\left\{v_{2} v_{3}, v_{4} v_{5}, \ldots, v_{2 s} v_{1}\right\}$ and $H=H^{\prime}$. Note that both $T_{1}$ and $T_{2}$ are forests. Since $d_{G}(x)=d_{G^{\prime}}(x)+2$ for vertices $x$ of the cycle $C$, we see that $d_{T_{1}}\left(v_{j}\right)=d_{T_{2}}\left(v_{j}\right)=1=\frac{d_{G}\left(v_{j}\right)}{2}$ for $j=1,3, \ldots, 2 s-1$, and $d_{T_{i}}\left(v_{j}\right)=$ $d_{T_{i}^{\prime}}\left(v_{j}\right)+1 \leq\left\lceil\frac{d_{G^{\prime}}\left(v_{j}\right)}{2}\right\rceil+1=\left\lceil\frac{d_{G}\left(v_{j}\right)}{2}\right\rceil$ for $i=1,2$ and $j=2,4, \ldots, 2 s$, the lemma holds.

The following is a direct consequence of Lemma 2 .

Corollary 3. Every planar graph $G$ without adjacent 3-cycles can be edgepartitioned into two forests $T_{1}, T_{2}$ and a subgraph $H$ such that $\Delta\left(T_{1}\right) \leq\left\lceil\frac{\Delta(G)}{2}\right\rceil$, $\Delta\left(T_{2}\right) \leq\left\lceil\frac{\Delta(G)}{2}\right\rceil$ and $\Delta(H) \leq 6$.

Lemma 4. If a graph $G$ can be edge-partitioned into $m$ subgraphs $G_{1}, G_{2}, \ldots$, $G_{m}$, then $l a_{2}(G) \leq \sum_{i=1}^{m} l a_{2}\left(G_{i}\right)$.

The above lemma is obvious since we just need to use disjoint color sets on the $G_{i}$ 's.

Lemma $5([5])$. For a forest $T$, we have $l a_{2}(T) \leq\left\lceil\frac{\Delta(T)+1}{2}\right\rceil$.

Lemma $6([2])$. For a graph $G$, we have la $a_{2}(G) \leq \Delta(G)$.

Now we are ready to prove our first main result.

Theorem 7. If $G$ is a planar graph without adjacent 3-cycles, then $l_{2}(G) \leq$ $\left\lceil\frac{\Delta(G)}{2}\right\rceil+8$.

Proof. By Corollary 3, $G$ has an edge-partition into two forests $T_{1}, T_{2}$ and a subgraph $H$ such that $\Delta\left(T_{1}\right) \leq\left\lceil\frac{\Delta(G)}{2}\right\rceil, \Delta\left(T_{2}\right) \leq\left\lceil\frac{\Delta(G)}{2}\right\rceil$ and $\Delta(H) \leq 6$. Combining Lemmas $4,5,6$, we obtain the following sequence of inequalities.

$$
\begin{aligned}
l a_{2}(G) & \leq l a_{2}\left(T_{1}\right)+l a_{2}\left(T_{2}\right)+l a_{2}(H) \\
& \leq\left\lceil\frac{\Delta\left(T_{1}\right)+1}{2}\right\rceil+\left\lceil\frac{\Delta\left(T_{2}\right)+1}{2}\right\rceil+\Delta(H) \\
& \leq 2\left\lceil\frac{\left\lceil\frac{\Delta(G)}{2}\right\rceil+1}{2}\right\rceil+6 \\
& \leq\left(\left\lceil\frac{\Delta(G)}{2}\right\rceil+2\right)+6 \\
& =\left\lceil\frac{\Delta(G)}{2}\right\rceil+8 .
\end{aligned}
$$


Lemma 8. Let $G$ be a connected planar graph with $\delta(G) \geq 2$. If $G$ has no adjacent 4-cycles, then $G$ contains an edge $x y$ such that $d(x)+d(y) \leq 13$, or $G$ contains a 2-alternating cycle.

Proof. Suppose, to the contrary, that $G$ is such a connected planar graph not satisfying the lemma. Then we have

(a) For any vertex $v, q_{3}(v) \leq\left\lfloor\frac{2 d(v)}{3}\right\rfloor$;

(b) For any vertex $v, n_{2}(v)+n_{3}(v)+\left\lceil\frac{q_{3}(v)}{2}\right\rceil \leq d(v)$;

(c) Let $G_{2}$ be the subgraph induced by the edges incident with the 2 -vertices of $G$, then $G_{2}$ is a forest and there exists a matching $M$ such that all 2-vertices in $G_{2}$ are saturated.

(a) is obvious. For (b), suppose $f$ is a 3 -face incident with $v$. Since $d(x)+$ $d(y) \geq 14$ for any edge $x y \in E(G), f$ is incident with at most one $6^{-}$-vertex. So $v$ is adjacent to at least $\left\lceil\frac{q_{3}(v)}{2}\right\rceil 7^{+}$-vertices. Hence, $d(v)-n_{2}(v)-n_{3}(v) \geq$ $d(v)-\sum_{i=2}^{6} n_{i}(v) \geq\left\lceil\frac{q_{3}(v)}{2}\right\rceil$.

For (c), it is similar to that of Lemma 1(c).

If $u v \in M$ and $d(u)=2$, we call $v$ the 2-master of $u$.

By Euler's formula $|V|-|E|+|F|=2$, we have

$$
\sum_{v \in V}(d(v)-4)+\sum_{f \in F}(d(f)-4)=-4(|V|-|E|+|F|)=-8<0 .
$$

We define $c h$ to be the initial charge. Let $\operatorname{ch}(v)=d(v)-4$ for each $v \in V(G)$ and $\operatorname{ch}(f)=d(f)-4$ for each $f \in F(G)$. In the following, we will reassign a new charge denoted by $\operatorname{ch}^{\prime}(x)$ to each $x \in V(G) \cup F(G)$ according to the discharging rules. Since our rules only move charges around, and do not affect the sum, we have

$$
\sum_{x \in V(G) \cup F(G)} \operatorname{ch}^{\prime}(x)=\sum_{x \in V(G) \cup F(G)} \operatorname{ch}(x)=-8 .
$$

In the following, we will show that $\operatorname{ch}^{\prime}(x) \geq 0$ for each $x \in V(G) \cup F(G)$, a contradiction to (4), completing the proof.

Now, let us introduce the needed discharging rules as follows:

R2-1. Each 2-vertex receives 2 from its 2-master.

R2-2. Each 3 -vertex receives $\frac{4}{15}$ from each of its neighbors.

R2-3. If a vertex $v$ is incident with a $5^{+}$-face $f$, then $v$ receives $\frac{1}{5}$ from $f$.

R2-4. Each 3 -face receives $\frac{1}{2}$ from each of its incident $7^{+}$-vertices.

Let $f$ be a face of $G$. If $d(f) \geq 5$, then $\operatorname{ch}^{\prime}(f) \geq \operatorname{ch}(f)-d(f) \times \frac{1}{5} \geq 0$ by R2-3. If $d(f)=4$, then $\operatorname{ch}^{\prime}(f)=\operatorname{ch}(f)=d(f)-4=0$. If $d(f)=3$, then it is incident with at least two $7^{+}$-vertices. It follows that $\operatorname{ch}^{\prime}(f) \geq \operatorname{ch}(f)+2 \times \frac{1}{2}=0$ by $\mathrm{R} 2-4$. 
Let $v$ be a vertex of $G$. If $d(v)=2$, then $\operatorname{ch}^{\prime}(v)=\operatorname{ch}(v)+2=0$ by R2-1. If $d(v)=3$, then $v$ is incident with at least one $5^{+}$-face and $\operatorname{ch}^{\prime}(v) \geq$ $\operatorname{ch}(v)+\frac{1}{5}+3 \times \frac{4}{15}=0$ by R2-2 and R2-3. If $4 \leq d(v) \leq 6$, then $\operatorname{ch}^{\prime}(v)=\operatorname{ch}(v)=$ $d(v)-4 \geq 0$. If $7 \leq d(v) \leq 10$, then $v$ is incident with at most $\left\lfloor\frac{2 d(v)}{3}\right\rfloor 3$-faces by (a), it follows that $\operatorname{ch}^{\prime}(v) \geq \operatorname{ch}(v)-\frac{1}{2}\left\lfloor\frac{2 d(v)}{3}\right\rfloor>0$ by R2-4. If $d(v)=11$, then $q_{3}(v) \leq 7$ by $(\mathrm{a})$, and $n_{3}(v) \leq d(v)-\left\lceil\frac{q_{3}(v)}{2}\right\rceil$ by (b). It follows that $\operatorname{ch}^{\prime}(v) \geq \operatorname{ch}(v)-\frac{1}{2} q_{3}(v)-\frac{4}{15} n_{3}(v)>0$ by R2-2 and R2-4. If $d(v) \geq 12$, then $q_{3}(v) \leq\left\lfloor\frac{2 d(v)}{3}\right\rfloor$ by $(\mathrm{a})$, and $n_{3}(v) \leq d(v)-n_{2}(v)-\left\lceil\frac{q_{3}(v)}{2}\right\rceil$ by (b). It follows that $\operatorname{ch}^{\prime}(v) \geq \operatorname{ch}(v)-\max \left\{2+\frac{1}{2} q_{3}(v)+\frac{4}{15}\left(d(v)-n_{2}(v)-\left\lceil\frac{q_{3}(v)}{2}\right\rceil\right), \frac{1}{2} q_{3}(v)+\right.$ $\frac{4}{15}\left(d(v)-\left\lceil\frac{q_{3}(v)}{2}\right\rceil\right) \geq \operatorname{ch}(v)-\max \left\{2+\frac{1}{2} q_{3}(v)+\frac{4}{15}\left(d(v)-1-\left\lceil\frac{q_{3}(v)}{2}\right\rceil\right), \frac{1}{2} q_{3}(v)+\right.$ $\frac{4}{15}\left(d(v)-\left\lceil\frac{q_{3}(v)}{2}\right\rceil\right) \geq 0$ by R2-1, R2-2 and R2-4.

Hence, we complete the proof of the lemma.

Using Lemma 8, the next result can be proved analogously to Lemma 2 .

Lemma 9. Every planar graph $G$ without adjacent 4-cycles can be edge-partitioned into two forests $T_{1}, T_{2}$ and a subgraph $H$ such that $\Delta\left(T_{1}\right) \leq\left\lceil\frac{\Delta(G)}{2}\right\rceil$, $\Delta\left(T_{2}\right) \leq\left\lceil\frac{\Delta(G)}{2}\right\rceil$ and $\Delta(H) \leq 8$.

Our second main result is the following theorem.

Theorem 10. If $G$ is a planar graph without adjacent 4 -cycles, then $l a_{2}(G) \leq$ $\left\lceil\frac{\Delta(G)}{2}\right\rceil+10$

Proof. We can prove it using an argument similar to the proof of Theorem 7 .

Lemma 11. Let $G$ be a connected planar graph with $\delta(G) \geq 2$. If any 3cycle is not adjacent to a 4-cycle of $G$, then $G$ contains an edge xy such that $d(x)+d(y) \leq 9$, or $G$ contains a 2-alternating cycle.

Proof. Suppose, to the contrary, that $G$ is such a connected planar graph not satisfying the lemma. Then we have

(a) Any 3 -face is not adjacent to a 3 -face;

(b) For any vertex $v, q_{3}(v) \leq\left\lfloor\frac{d(v)}{2}\right\rfloor$;

(c) Let $G_{2}$ be the subgraph induced by the edges incident with the 2-vertices of $G$, then $G_{2}$ is a forest and there exists a matching $M$ such that all 2-vertices in $G_{2}$ are saturated.

By Euler's formula $|V|-|E|+|F|=2$, we have

$$
\sum_{v \in V}(2 d(v)-6)+\sum_{f \in F}(d(f)-6)=-6(|V|-|E|+|F|)=-12<0 .
$$

We define $c h$ to be the initial charge. Let $\operatorname{ch}(v)=2 d(v)-6$ for each $v \in V(G)$ and $\operatorname{ch}(f)=d(f)-6$ for each $f \in F(G)$. In the following, we will reassign 
a new charge denoted by $\operatorname{ch}^{\prime}(x)$ to each $x \in V(G) \cup F(G)$ according to the discharging rules. Since our rules only move charges around, and do not affect the sum, we have

$$
\sum_{x \in V(G) \cup F(G)} \operatorname{ch}^{\prime}(x)=\sum_{x \in V(G) \cup F(G)} \operatorname{ch}(x)=-12 .
$$

In the following, we will show that $\operatorname{ch}^{\prime}(x) \geq 0$ for each $x \in V(G) \cup F(G)$, a contradiction to (6), completing the proof.

The discharging rules are defined as follows.

R3-1. Each 2-vertex receives 2 from its 2-master.

R3-2. Each 5 -vertex sends 1 to each of its incident 3 -faces, $\frac{1}{2}$ to each of its incident other faces.

R3-3. Each $6^{+}$-vertex sends $\frac{3}{2}$ to each of its incident 3 -faces, 1 to each of its incident 4 -faces, $\frac{1}{3}$ to each of its incident 5 -faces.

In particular, we have

Remark 1 . Let $d(v) \geq 6, f_{1}, f_{2}, \ldots, f_{d}$ be the faces incident with $v$ in a clockwise order. If $d\left(f_{i}\right)=3$, then $d\left(f_{i+1}\right) \geq 5$. $v$ sends at most $\frac{3}{2}+\frac{1}{3}=\frac{11}{6}$ to $f_{i}$ and $f_{i+1}$; If $d\left(f_{i}\right)=d\left(f_{i+1}\right)=4$, then $v$ sends 2 to $f_{i}$ and $f_{i+1}$.

Let $f$ be a face of $G$. If $d(f) \geq 6$, then $\operatorname{ch}^{\prime}(f)=c h(f) \geq 0$. If $d(f)=5$, then it is incident with at most two $4^{-}$-vertices. If $f$ is incident with two $4^{-}$-vertices, then the other three vertices must be $6^{+}$-vertices. It follows that $\operatorname{ch}^{\prime}(f) \geq \operatorname{ch}(f)+3 \times \frac{1}{3}=0$ by R3-3. If $f$ is incident with one $4^{-}$-vertices, then $\operatorname{ch}^{\prime}(f) \geq \operatorname{ch}(f)+4 \times \frac{1}{3}>0$ by R3-2 and R3-3. If $f$ is not incident with any $4^{-}$-vertices, then $\operatorname{ch}^{\prime}(f) \geq \operatorname{ch}(f)+5 \times \frac{1}{3}>0$ by R3-2 and R3-3. If $d(f)=4$, then it is incident with at most two $4^{-}$-vertices. If $f$ is incident with at least one $4^{-}$-vertex, then $f$ is incident with at least two $6^{+}$-vertices. Hence, $\operatorname{ch}^{\prime}(f) \geq \operatorname{ch}(f)+2 \times 1=0$ by R3-3. If $f$ is not incident with $4^{-}$-vertices, then $f$ receives at least $\frac{1}{2}$ from each of its incident vertices by R3-2 and R3-3. Hence, $\operatorname{ch}^{\prime}(f) \geq \operatorname{ch}(f)+4 \times \frac{1}{2}=0$. If $d(f)=3$, then it is incident with at most one $4^{-}$-vertex. If $f$ is incident with one $4^{-}$-vertex, then the other two vertices must be $6^{+}$-vertices. Hence, $\operatorname{ch}^{\prime}(f) \geq \operatorname{ch}(f)+2 \times \frac{3}{2}=0$ by R3-3. Otherwise, $f$ receives at least 1 from each of its incident vertices by $\mathrm{R} 3-2$ and $\mathrm{R} 3-3$. It follows that $\operatorname{ch}^{\prime}(f) \geq \operatorname{ch}(f)+3 \times 1=0$.

Let $v$ be a vertex of $G$. If $d(v)=2$, then $\operatorname{ch}^{\prime}(v)=\operatorname{ch}(v)+2=0$ by R3-1. If $3 \leq d(v) \leq 4$, then $\operatorname{ch}^{\prime}(v)=\operatorname{ch}(v) \geq 0$. If $d(v)=5$, then $v$ is incident with at most two 3 -faces. It follows that $\operatorname{ch}^{\prime}(v) \geq \operatorname{ch}(v)-2 \times 1-3 \times \frac{1}{2}>0$ by R3-2.

By Remark 1 , for $d(v) \geq 6$, we only need to consider the case that $v$ is incident with $d(v) 4$-faces.

If $6 \leq d(v) \leq 7$, then $\operatorname{ch}^{\prime}(v) \geq \operatorname{ch}(v)-d(v) \times 1 \geq 0$ by R3-3. If $d(v) \geq 8$, then $\operatorname{ch}^{\prime}(v) \geq \operatorname{ch}(v)-2-d(v) \times 1 \geq 0$ by R3-1 and R3-3.

Hence we complete the proof of the lemma. 
Using Lemma 11, the next result can be proved analogously to Lemma 2.

Lemma 12. Let $G$ be a planar graph. If any 3-cycle is not adjacent to a 4cycle, then $G$ has an edge-partition into two forests $T_{1}, T_{2}$ and a subgraph $H$ such that $\Delta\left(T_{1}\right) \leq\left\lceil\frac{\Delta(G)}{2}\right\rceil, \Delta\left(T_{2}\right) \leq\left\lceil\frac{\Delta(G)}{2}\right\rceil$ and $\Delta(H) \leq 4$.

Our third main result is the following theorem.

Theorem 13. If $G$ is a planar graph that any 3-cycle is not adjacent to a 4-cycle, then $l a_{2}(G) \leq\left\lceil\frac{\Delta(G)}{2}\right\rceil+6$.

The proof of Theorem 13 is similar to that of Theorem 7, we omit here.

\section{References}

[1] R. E. L. Aldred and N. C. Wormald, More on the linear $k$-arboricity of regular graphs, Australas. J. Combin. 18 (1998), 97-104.

[2] J. C. Bermond, J. L. Fouquet, M. Habib, and B. Péroche, On linear k-arboricity, Discrete Math. 52 (1984), no. 2-3, 123-132.

[3] G. J. Chang, Algorithmic aspects of linear k-arboricity, Taiwanese J. Math. 3 (1999), no. $1,73-81$.

[4] G. J. Chang, B. L. Chen, H. L. Fu, and K. C. Huang, Linear k-arboricity on trees, Discrete Appl. Math. 103 (2000), no. 1-3, 281-287.

[5] B. L. Chen, H. L. Fu, and K. C. Huang, Decomposing graphs into forests of paths with size less than three, Australas. J. Combin. 3 (1991), 55-73.

[6] H. L. Fu and K. C. Huang, The linear 2-arboricity of complete bipartite graphs, Ars Combin. 38 (1994), 309-318.

[7] M. Habib and B. Péroche, Some problems about linear arboricity, Discrete Math. 41 (1982), no. 2, 219-220.

[8] B. Jackson and N. C. Wormald, On linear k-arboricity of cubic graphs, Discrete Math. 162 (1996), no. 1-3, 293-297.

[9] K. W. Lih, L. D. Tong, and W. F. Wang, The linear 2-arboricity of planar graphs, Graphs Combin. 19 (2003), no. 2, 241-248.

[10] Q. Ma and J. L. Wu, Planar graphs without 5-cycles or without 6-cycles, Discrete Math. (2008), doi:10.1016/j.disc.2008.07.033.

[11] J. Qian and W. F. Wang, The linear 2-arboricity of planar graphs without 4-cycles, J. Zhejiang Norm. Univ. 29 (2006), no. 2, 121-125.

[12] C. Thomassen, Two-coloring the edges of a cubic graph such that each monochromatic component is a path of length at most 5, J. Combin. Theory Ser. B 75 (1999), no. 1, $100-109$.

HONG-Yu CHEN

SCHOOL OF SCIENCES

Shanghai Institute of Technology

Shanghai, 201418, P. R. China

E-mail address: hongyuchen86@163.com

XIANG TAN

School of Statistics and Mathematics

Shandong University of Finance

Jinan, Shandong, 250014, P. R. China

E-mail address: xtan@ruyi.com 
JiAN-LIANG WU

School of Mathematics

SHANDONG UNIVERSITY

Jinan, Shandong, 250100, P. R. China

E-mail address: jlwu@sdu.edu.cn 